\title{
On Corrected Quadrature Rules and Optimal Error Bounds
}

\author{
François Dubeau \\ Département de Mathématiques, Faculté des Sciences, Université de Sherbrooke, 2500 boulevard de l'Université, \\ Sherbrooke, QC, Canada J1K 2R1 \\ Correspondence should be addressed to François Dubeau; francois.dubeau@usherbrooke.ca
}

Received 25 February 2015; Accepted 13 May 2015

Academic Editor: Fasma Diele

Copyright ( 2015 François Dubeau. This is an open access article distributed under the Creative Commons Attribution License, which permits unrestricted use, distribution, and reproduction in any medium, provided the original work is properly cited.

\begin{abstract}
We present an analysis of corrected quadrature rules based on the method of undetermined coefficients and its associated degree of accuracy. The correcting terms use weighted values of the first derivative of the function at the endpoint of the subinterval in such a way that the composite rules contain only two new values. Using Taylor's expansions and Peano's kernels we obtain best truncation error bounds which depend on the regularity of the function and the weight parameter. We can minimize the bounds with respect to the parameter, and we can find the best parameter value to increase the order of the error bounds or, equivalently, the degree of accuracy of the rule.
\end{abstract}

\section{Introduction}

The problem of correcting known quadrature formulas by using derivative values is an old one (see [1] and the references therein) and continues regularly to be analyzed $[2,3]$. Here we propose an analysis of corrected quadrature rules using the method of undetermined coefficients and its associated degree of accuracy (or precision). Using Taylor's expansions for absolutely continuous functions, we obtain Peano's kernels and, as a consequence, best error bounds. The bounds, expressed in terms of $L^{p}$-norms, depend on the regularity of the function and also on a parameter $\beta$. For a given regularity of the function and a $p$-norm, we can minimize the bound with respect to $\beta$. Moreover, under certain conditions, it is possible to find a value $\beta_{*}$ of the parameter $\beta$ to increase the degree of accuracy (and the order of error bounds) of the method.

Let us start by considering the integral we want to approximate:

$$
Q(f ; h):=\frac{1}{h} \int_{-h}^{h} f(x) d x .
$$

This form of the integral is motivated by the composite quadrature formula considered in Section 7 which consists in adding terms of the form $h Q(f ; h)$. The expression $h Q(f ; h)$ also represents any definite integral subject to a linear transformation of the argument.
The method of undetermined coefficients consists in finding a $(n+1)$-dimensional weight vector $\vec{a}=\left(a_{0}, \ldots, a_{n}\right)$ associated with a given $(n+1)$-dimensional vector of distinct coordinates (or nodes) $\vec{x}=\left(x_{0}, \ldots, x_{n}\right)$ such that $Q(f ; h)$ is approximated by the finite sum

$$
Q(f ; h) \approx Q_{n}(f ; h):=\sum_{i=0}^{n} a_{i} f\left(h x_{i}\right) .
$$

In this expression we use $h x_{i}$ to fix the position of the nodes relatively to the interval $[-h, h]$ for any $h$.

For weighted endpoint corrected quadrature rules, the approximation of (1) is given by

$$
\begin{aligned}
\widetilde{Q}_{n}(f ; h, \beta):= & \sum_{i=0}^{n} a_{i}(\beta) f\left(h x_{i}\right) \\
& +\beta h\left[f^{(1)}(h)-f^{(1)}(-h)\right] .
\end{aligned}
$$

The endpoint correction term is

$$
Q^{c}(f ; h):=h\left[f^{(1)}(h)-f^{(1)}(-h)\right],
$$

and $\beta$ is the weight or the parameter. In (3), the weights $a_{i}$ 's depend on $\beta$. The truncation error of the process is

$$
R_{n}(f ; h, \beta):=Q(f ; h)-\widetilde{Q}_{n}(f ; h, \beta),
$$


and the method is based on the requirement that

$$
R_{n}(f ; h, \beta)=o\left(h^{n}\right),
$$

for a regular enough function.

The plan of the paper is the following. In the next section we present preliminaries about polynomials and Taylor's expansions. The method of undetermined coefficients adapted to include correction terms is the object of Section 3. In Section 4 we obtain optimal error bounds using Taylor's expansions and Peano's kernels. In Section 5 we present the way we can choose the parameter $\beta$ to increase the degree of accuracy and the order of the error bound of the method. Examples are given in Section 6. Composite rules are presented in Section 7 with numerical examples.

Throughout this paper we will use $f^{(l)}(x)$ for the $l$ th derivative of $f(x)$ for $l=1,2, \ldots$. Let $1 \leq p \leq \infty$; if $f(x)$ is defined on a set $E,\|f\|_{p, E}$ will be its $L^{p}$-norm on $E$, and if $\vec{v}$ is a vector in $\mathbb{R}^{n}$, its $l^{p}$-norm will be $\|\vec{v}\|_{p}$.

\section{Preliminaries}

2.1. Small o and Big O Notations. Let $f(x)$ be a function such that $\lim _{x \rightarrow \alpha} f(x)=0$. We say that $g(x)$ is small $o$ of $f(x)$ around $\alpha$ and write $g(x)=o(f(x))$, if for any $\epsilon>0$ there exists a $\delta_{\epsilon}>0$ such that

$$
|g(x)| \leq \epsilon|f(x)|
$$

which holds for $0<|x-\alpha|<\delta_{\epsilon}$. We say that $g(x)$ is big $O$ of $f(x)$ around $\alpha$ and write $g(x)=O(f(x))$, if there exist a constant $C$ and a $\delta>0$ such that

$$
|g(x)| \leq C|f(x)|
$$

which holds for $0<|x-\alpha|<\delta$.

2.2. Polynomials and Small o Notation. The next lemma is a direct consequence of the small $o$ notation for polynomials and will be useful to obtain necessary conditions in Section 4 .

Lemma 1. Let $r$ be a positive real number and $n=\lfloor r\rfloor$. Let $\pi_{m}(x)$ be a polynomial of degree $m$ such that

$$
\pi_{m}(x)=o\left(|x-\alpha|^{r}\right) .
$$

Then,

$$
\pi_{m}(x)= \begin{cases}(x-\alpha)^{n+1} \pi_{m-(n+1)}(x) & \text { if } m>r \\ 0 & \text { if } m \leq r\end{cases}
$$

where $\pi_{m-(n+1)}(x)$ is a polynomial of degree $m-(n+1)$.

2.3. Taylor's Expansion. Let $I_{h}=[-h, h]$; for $h=1$ we will simply use $I=[-1,1], I_{h}^{+}=[0, h]$, and $I_{h}^{-}=[-h, 0]$; for $h=1$ we will simply use $I^{+}=[0,1]$ and $I^{-}=[-1,0]$. Let $p$ and $q$ be two extended real numbers such that $1 \leq p, q \leq \infty$, and $1 / p+1 / q=1$. Let $C^{l}\left(I_{h}\right)$ be the set of continuously differentiable functions up to order $l$ on $I_{h}$. Let $A C^{l+1, p}\left(I_{h}\right)$ be the set of absolutely continuous functions on $I_{h}$ be defined by $f \in A C^{l+1, p}\left(I_{h}\right)$ if and only if

$$
f \in C^{l}\left(I_{h}\right)
$$

and
(a) $f^{(l+1)} \in L^{p}\left(I_{h}\right)$,
(b) $f^{(l)}(s)=f^{(l)}(r)+\int_{r}^{s} f^{(l+1)}(\xi) d \xi$, for all $r, s \in I_{h}$.

Taylor's expansion of $f(x) \in A C^{l+1, p}\left(I_{h}\right)$ around $x=0$ of order $l+1$ is

$$
f(x)=\sum_{j=0}^{l} \frac{f^{(j)}(0)}{j !} x^{j}+\int_{-h}^{h} f^{(l+1)}(y) K_{T, l}(x, y ; h) d y,
$$

where $K_{T, l}(x, y ; h)$ is the kernel

$$
\begin{gathered}
K_{T, l}(x, y ; h)=\frac{1}{l !}\left[(x-y)_{+}^{l} \mathbf{1}_{[0, h]}(y)\right. \\
\left.+(-1)^{l+1}(y-x)_{+}^{l} \mathbf{1}_{[-h, 0]}(y)\right],
\end{gathered}
$$

for any $x, y$ in $I_{h}$ (see $\left.[4,5]\right)$. This kernel is a piecewise polynomial function of degree $l$. In this expression, if $E$ is a set, then

$$
\mathbf{1}_{E}(y)= \begin{cases}1 & \text { if } y \in E, \\ 0 & \text { if } y \notin E,\end{cases}
$$

and, for any nonnegative integer $l$,

$$
(z)_{+}^{l}= \begin{cases}z^{l} & \text { if } z>0 \\ 0 & \text { if } z \leq 0\end{cases}
$$

If we set $x=h \xi$ and $y=h \eta$, then the kernel becomes

$$
K_{T, l}(x, y ; h)=K_{T, l}(h \xi, h \eta ; h)=h^{l} K_{T, l}(\xi, \eta ; 1)
$$

for any $\xi, \eta$ in $I$.

\section{The Method of Undetermined Coefficients}

3.1. Direct Approach. We observe that $R_{n}(f ; h, \beta)$ given by (5) is linear with respect to $f(x)$, and if $f(x)$ is a polynomial of degree $\leq m$ with respect to $x$, then $R_{n}(f ; h, \beta)$ is a polynomial of degree $\leq m$ with respect to $h$. From Lemma 1, condition (6) implies that $R_{n}(f ; h, \beta)=0$ for any polynomial $f(x)$ of degree $\leq n$. Then using the standard basis $\left\{x^{l}\right\}_{l=0}^{n}$, we have to solve the linear system

$$
V(\vec{x}) \vec{a}(\beta)=\vec{b}(\beta),
$$

where $V(\vec{x})$ is the Vandermonde matrix associated with $\vec{x}$, and the entries of $\vec{b}(\beta)$ are

$$
b_{l}(\beta)=\left[\frac{1}{l+1}-l \beta\right]\left(1+(-1)^{l}\right) \quad \text { for } l=0, \ldots, n .
$$


The solution of this system is

$$
\begin{aligned}
\vec{a}(\beta) & =\sum_{l=0}^{n}\left[\frac{1}{l+1}-l \beta\right]\left(1+(-1)^{l}\right) V^{-1}(\vec{x}) \vec{e}_{l} \\
& =\sum_{k=0}^{\lfloor n / 2\rfloor} 2\left[\frac{1}{2 k+1}-2 k \beta\right] V^{-1}(\vec{x}) \vec{e}_{2 k} \\
& =\sum_{k=0}^{\lfloor n / 2\rfloor} \frac{2}{2 k+1} V^{-1}(\vec{x}) \vec{e}_{2 k}-\beta \sum_{k=0}^{\lfloor n / 2\rfloor} 4 k V^{-1}(\vec{x}) \vec{e}_{2 k},
\end{aligned}
$$

where $\vec{e}_{l}$ is the $(n+1)$-column vector, the transpose of $\left(\delta_{l, 0}, \ldots, \delta_{l, j} \ldots, \delta_{l, n}\right)$, and $\delta_{l, j}=1$ if $j=l, \delta_{l, j}=0$ if $j \neq l$, for $0 \leq l, j \leq n$. Let us remark that $\vec{a}(\beta)$ does not depend on $\beta$ for $n=0$ and $n=1$ (cases which include the midpoint and trapezoidal rules).

It might happen that $R_{n}(f ; h, \beta)=0$ for some polynomials $f(x)$ of degree $>n$. Let us define the degree of accuracy (or precision) $n_{\beta}$ of the approximation process (3) as the largest integer $n_{\beta} \geq n$ such that $R_{n}(f ; h, \beta)=0$ holds for any polynomial $f(x)$ of degree $l \leq n_{\beta}$.

3.2. A Decomposition. Using (19), let us set

$$
\begin{aligned}
\vec{a} & =\sum_{k=0}^{\lfloor n / 2\rfloor} \frac{2}{2 k+1} V^{-1}(\vec{x}) \vec{e}_{2 k}, \\
\vec{a}^{c} & =\sum_{k=0}^{\lfloor n / 2\rfloor} 4 k V^{-1}(\vec{x}) \vec{e}_{2 k} .
\end{aligned}
$$

Then $\vec{a}$ is the solution of

$$
V(\vec{x}) \vec{a}=\vec{b},
$$

where the entries of $\vec{b}$ are

$$
b_{l}=\frac{\left(1+(-1)^{l}\right)}{l+1} \text { for } l=0, \ldots, n,
$$

and $\vec{a}^{c}$ is the solution of

$$
V(\vec{x}) \vec{a}^{c}=\vec{b}^{c},
$$

where the entries of $\vec{b}^{c}$ are

$$
b_{l}^{c}=l\left(1+(-1)^{l}\right) \quad \text { for } l=0, \ldots, n .
$$

Let us write

$$
\begin{aligned}
& R_{n}(f ; h)=Q(f ; h)-\sum_{i=0}^{n} a_{i} f\left(h x_{i}\right), \\
& R_{n}^{c}(f ; h)=Q^{c}(f ; h)-\sum_{i=0}^{n} a_{i}^{c} f\left(h x_{i}\right) .
\end{aligned}
$$

Then the following result follows directly.
Theorem 2. The following two conditions are equivalent:

(A) for any $\beta$, there exists a unique $\vec{a}(\beta)$ such that $R_{n}(f$; $h, \beta)=0$ for any polynomial of degree $\leq n$,

(B) there exists a unique $\vec{a}$ such that $R_{n}(f ; h)=0$ for any polynomial of degree $\leq n$, and there exists a unique $\vec{a}^{c}$ such that $R_{n}^{c}(f ; h)=0$ for any polynomial of degree $\leq n$.

Moreover, one has $\vec{a}(\beta)=\vec{a}-\beta \vec{a}^{c}$ and

$$
R_{n}(f ; h, \beta)=R_{n}(f ; h)-\beta R_{n}^{c}(f ; h) .
$$

\section{Truncation Error}

Since the method requires the values of $f^{(1)}(-h)$ and $f^{(1)}(h)$ for $\beta \neq 0$, we assume that $f(x)$ is at least in $A C^{2, p}\left(I_{h}\right)$. Let $l$ such that $1 \leq l \leq n_{\beta}$ and $f(x) \in A C^{l+1, p}\left(I_{h}\right)$. Since the process is exact for polynomials of degree $\leq l$, using a Taylor expansion (12) of order $l+1$, we obtain

$$
R_{n}(f ; h, \beta)=\int_{-h}^{h} f^{(l+1)}(y) K_{\mathrm{Q}, l}(y ; h, \beta) d y,
$$

where $K_{\mathrm{Q}, l}(y ; h, \beta)$ is the Peano kernel given by

$$
K_{\mathrm{Q}, l}(y ; h, \beta)=R_{n}\left(K_{T, l}(\cdot, y ; h) ; h, \beta\right) .
$$

Following (26), we have

$$
K_{\mathrm{Q}, l}(y ; h, \beta)=K_{\mathrm{Q}, l}(y ; h)-\beta K_{\mathrm{Q}^{c}, l}(y ; h),
$$

where

$$
\begin{aligned}
K_{\mathrm{Q}, l}(y ; h)= & R_{n}\left(K_{T, l}(\cdot, y ; h) ; h\right) \\
= & \frac{1}{h} \int_{-h}^{h} K_{T, l}(x, y ; h) d x \\
& -\sum_{i=0}^{n} a_{i} K_{T, l}\left(h x_{i}, y ; h\right), \\
K_{Q^{c}, l}(y ; h)= & R_{n}^{c}\left(K_{T, l}(\cdot, y ; h) ; h\right) \\
= & h\left[K_{T, l-1}(h, y ; h)-K_{T, l-1}(-h, y ; h)\right] \\
& -\sum_{i=0}^{n} a_{i}^{c} K_{T, l}\left(h x_{i}, y ; h\right),
\end{aligned}
$$

since $K_{T, l}^{(1)}(x, y ; h)=K_{T, l-1}(x, y ; h)$. We observe that

$$
\begin{aligned}
K_{\mathrm{Q}, l}(y ; h, \beta) & =K_{\mathrm{Q}, l}(h \eta ; h, \beta)=h^{l} K_{\mathrm{Q}, l}(\eta ; 1, \beta) \\
& =h^{l}\left[K_{\mathrm{Q}, l}(\eta ; 1)-\beta K_{Q^{c}, l}(\eta ; 1)\right],
\end{aligned}
$$


where

$$
\begin{aligned}
& K_{\mathrm{Q}, l}(\eta ; 1)=\frac{1}{(l+1) !}\left[(1-\eta)^{l+1} \mathbf{1}_{I^{+}}(\eta)+(-1)^{l+1}(\eta\right. \\
& \left.+1)^{l+1} \mathbf{1}_{I^{-}}(\eta)\right]-\frac{1}{l !} \sum_{i=0}^{n} a_{i}\left[\left(x_{i}-\eta\right)_{+}^{l} \mathbf{1}_{I^{+}}(\eta)\right. \\
& \left.+(-1)^{l+1}\left(\eta-x_{i}\right)_{+}^{l} \mathbf{1}_{I^{-}}(\eta)\right], \\
& K_{\mathrm{Q}^{c}, l}(\eta ; 1)=\frac{1}{(l-1) !}\left[(1-\eta)^{l-1} \mathbf{1}_{I^{+}}(\eta)+(-1)^{l+1}(\eta\right. \\
& \left.+1)^{l-1} \mathbf{1}_{I^{-}}(\eta)\right]-\frac{1}{l !} \sum_{i=0}^{n} a_{i}^{c}\left[\left(x_{i}-\eta\right)_{+}^{l} \mathbf{1}_{I^{+}}(\eta)\right. \\
& \left.\quad+(-1)^{l+1}\left(\eta-x_{i}\right)_{+}^{l} \mathbf{1}_{I^{-}}(\eta)\right] .
\end{aligned}
$$

So

$$
\begin{aligned}
\left\|K_{\mathrm{Q}, l}(\cdot ; h, \beta)\right\|_{q, I_{h}} & =h^{l+1-(1 / p)}\left\|K_{\mathrm{Q}, l}(\cdot ; 1, \beta)\right\|_{q, I}, \\
\left|R_{n}(f ; h, \beta)\right| & \leq h^{l+1-(1 / p)} C_{l, p}(\beta)\left\|f^{(l+1)}\right\|_{p, I_{h}},
\end{aligned}
$$

where

$$
\begin{aligned}
C_{l, p}(\beta) & =\left\|K_{Q, l}(\cdot ; 1, \beta)\right\|_{q, I} \\
& =\left\|K_{Q, l}(\cdot ; 1)-\beta K_{Q^{c}, l}(\cdot ; 1)\right\|_{q, I}
\end{aligned}
$$

does not depend on $h$. Since we have

$$
\lim _{h \rightarrow 0}\left\|f^{(l+1)}\right\|_{p, I_{h}}= \begin{cases}0 & \text { for } 1 \leq p<\infty, \\ C & \text { for } p=\infty,\end{cases}
$$

this result says that

$$
R_{n}(f ; h, \beta)= \begin{cases}o\left(h^{l+1-(1 / p)}\right) & \text { for } 1 \leq p<\infty, \\ O\left(h^{l+1}\right) & \text { for } p=\infty\end{cases}
$$

Since an $o\left(h^{l+1-(1 / p)}\right)$ or an $O\left(h^{l+1}\right)$ is an $o\left(h^{l}\right)$, (37) means that $R_{n}(f ; h, \beta)=o\left(h^{l}\right)$. In summary we have proved the following theorem which presents not only a sufficient condition but also a necessary condition to obtain the desired error order.

Theorem 3. A necessary and sufficient condition to have $R_{n}(f$; $h, \beta)=o\left(h^{n}\right)$ for any $f \in A C^{n+1, p}\left(I_{h}\right)$ is that $R_{n}(f ; h, \beta)=0$ for any polynomial $f(x)$ of degree $\leq n$.

As an extension of this result, the next one shows the dependance of the error in terms of the regularity $l$ of the function and the $p$-norm of $f^{(l+1)}(x)$.

Theorem 4. If $R_{n}(f ; h, \beta)=0$ for any polynomial $f(x)$ of degree $\leq n_{\beta}$, then (34), (35), and (37) hold for any $f \in$ $A C^{l+1, p}\left(I_{h}\right)$ and $1 \leq l \leq n_{\beta}$.
From (35), for fixed $l$ and $p, C_{l, p}(\beta)$ is a continuous function with respect to $\beta$. Moreover

$$
\lim _{|\beta| \rightarrow+\infty} C_{l, p}(\beta)=\lim _{|\beta| \rightarrow+\infty}\left\|K_{Q, l}(\cdot ; 1, \beta)\right\|_{q, I}=+\infty ;
$$

then we have the following result.

Theorem 5. For any given $l$ and $p$ (then $q$ is also given), the constant $C_{l, p}(\beta)$ reaches its minimum value with respect to $\beta$.

Let us set

$$
\beta_{q}^{*}=\arg \min _{\beta \in \mathbb{R}} C_{l, p}(\beta)=\arg \min _{\beta \in \mathbb{R}}\left\|K_{\mathrm{Q}, l}(\cdot ; 1, \beta)\right\|_{q, I} ;
$$

then

$$
C_{l, p}\left(\beta_{q}^{*}\right) \leq C_{l, p}(\beta)
$$

for all $\beta$.

Remark 6. It can be shown that bounds given by (34) and (35) are best bounds. Indeed, using a standard construction [6], we can find a function $f$, which depends on $l$ and $p$ (then $q$ is given), such that

$$
\begin{aligned}
R_{n}(f ; h, \beta) & =\left\|K_{\mathrm{Q}, l}(\cdot ; h, \beta)\right\|_{q, I_{h}}\left\|f^{(l+1)}\right\|_{p, I_{h}} \\
& =h^{l+1-(1 / p)} C_{l, p}(\beta)\left\|f^{(l+1)}\right\|_{p, I_{h}} .
\end{aligned}
$$

\section{Increasing the Degree of Accuracy and the Order of Error Bounds}

To increase the degree of accuracy of the process and consequently increase the order of error bounds, we consider the decomposition (26). Then we have the following result.

Theorem 7. Let

(i) $n_{\beta} \geq n$ be the degree of accuracy of $R_{n}(f ; h, \beta)$;

(ii) $n_{0} \geq n$ be the degree of accuracy of $R_{n}(f ; h)$;

(iii) $n_{c} \geq n$ be the degree of accuracy of $R_{n}^{c}(f ; h)$.

One can increase the degree of accuracy of $R_{n}(f ; h, \beta)$ if and only if $n_{0}=n_{c}$, for

$$
\beta=\beta_{*}=\frac{R_{n}\left(x^{n_{c}+1} ; h\right)}{R_{n}^{c}\left(x^{n_{c}+1} ; h\right)}=\frac{R_{n}\left(x^{n_{c}+1} ; 1\right)}{R_{n}^{c}\left(x^{n_{c}+1} ; 1\right)} .
$$

Proof. Using (26), we observe that $n_{\beta} \geq \min \left\{n_{0}, n_{c}\right\}$. So the parameter $\beta$ can be used to increase the degree of accuracy of the formula as follows:

(i) if $n_{0} \geq n_{c}$ : let

$$
\beta_{*}=\frac{R_{n}\left(x^{n_{c}+1} ; h\right)}{R_{n}^{c}\left(x^{n_{c}+1} ; h\right)}=\frac{R_{n}\left(x^{n_{c}+1} ; 1\right)}{R_{n}^{c}\left(x^{n_{c}+1} ; 1\right)} ;
$$

(a) if $n_{0}>n_{c}$, since $R_{n}\left(x^{n_{c}+1} ; 1\right)=0$, then $\beta_{*}=0$, $n_{\beta_{*}}=n_{0}$, and $n_{\beta}=n_{c}<n_{0}$ for any $\beta \neq \beta_{*}=0$, so we cannot increase the degree of accuracy; 
(b) if $n_{0}=n_{c}$, then $\beta_{*} \neq 0, n_{\beta_{*}}>n_{0}=n_{c}$, so we can increase of the degree of accuracy;

(ii) if $n_{0}<n_{c}$, then $n_{\beta}=n_{0}<n_{c}$ for any $\beta$, so we cannot increase the degree of accuracy.

So the result follows.

\section{Examples}

In this section we analyze four corrected quadrature rules: the midpoint and trapezoidal rules and two Simpson's rules: $\vec{x}=$ $(-1,0,1)$ with $\vec{a}=(1 / 3,4 / 3,1 / 3)$ and $\vec{x}=(-1,-1 / 3,1 / 3,1)$ with $\vec{a}=(1 / 4,3 / 4,3 / 4,1 / 4)$. We identify $\beta_{*}$ such that $n_{\beta_{*}}>$ $n_{\beta}$ for all $\beta \in \mathbb{R} \backslash\left\{\beta_{*}\right\}$ when it exists. Moreover for the midpoint and trapezoidal rules we compute the best $\beta_{q}^{*}$ for $q=1,2,+\infty$ given by (39).
6.1. Midpoint Rule. Let $n=0$ and $\vec{x}=(0)$; then the quadrature formula is

$$
\int_{-1}^{1} f(x) d x \approx 2 f(0)+\beta\left[f^{(1)}(1)-f^{(1)}(-1)\right],
$$

where $\vec{a}=(2)$ with $n_{0}=1$ and $\vec{a}^{c}=(0)$ with $n_{c}=1$. For $1 \leq l \leq n_{\beta}$, the kernel is given by

$$
\begin{gathered}
K_{\mathrm{Q}, l}(\eta ; 1, \beta)=\frac{(1-\eta)^{l-1}}{(l+1) !}\left[(1-\eta)^{2}-l(l+1) \beta\right] \\
\cdot \mathbf{1}_{I^{+}}(\eta)+(-1)^{l+1} \\
\cdot \frac{(\eta+1)^{l-1}}{(l+1) !}\left[(\eta+1)^{2}-l(l+1) \beta\right] \mathbf{1}_{I^{-}}(\eta) .
\end{gathered}
$$

Then $C_{l, p}(\beta)=\left\|K_{Q, l}(\cdot ; 1, \beta)\right\|_{q, I}$, where

$$
\left\|K_{\mathrm{Q}, l}(\cdot ; 1, \beta)\right\|_{q, I}= \begin{cases}\frac{1}{(l+1) !}\left[2 \int_{0}^{1}\left[(1-\eta)^{l-1}\left|(1-\eta)^{2}-l(l+1) \beta\right|\right]^{q} d \eta\right]^{1 / q} & \text { for } 1 \leq q<\infty \\ \frac{1}{(l+1) !} \max _{\eta \in[0,1]}(1-\eta)^{l-1}\left|(1-\eta)^{2}-l(l+1) \beta\right| & \text { for } q=\infty .\end{cases}
$$

For this method, $R_{0}\left(x^{2} ; 1\right)=2 / 3$ and $R_{0}^{c}\left(x^{2} ; 1\right)=4$, so $\beta_{*}=1 / 6$. We obtain $n_{\beta_{*}}=3$, and for any $\beta \neq \beta_{*}$ we have $n_{\beta}=1$. We have computed some constants $C_{l, p}(\beta)$ with $p=$ $1,2,+\infty$ (or $q=+\infty, 2,1)$, for $l=1\left(=n_{\beta}\right)$ using $\beta=0 \neq \beta_{*}$ and $\beta_{q}^{*} \neq \beta_{*}$, and for $l=1,2,3\left(=n_{\beta_{*}}\right)$ using $\beta=\beta_{*}=1 / 6$ as reported in Table 1. In summary

(i) for $l=1$, we have $C_{1, p}\left(\beta_{q}^{*}\right) \leq C_{1, p}\left(\beta_{*}\right) \leq C_{1, p}(0)$;

(ii) for $\beta_{*}=1 / 6$, we have $C_{3, p}\left(\beta_{*}\right) \leq C_{2, p}\left(\beta_{*}\right) \leq C_{1, p}\left(\beta_{*}\right)$.

6.2. Trapezoidal Rule. Let $n=1$ and $\vec{x}=(-1,1)$; then the quadrature formula is

$$
\begin{aligned}
\int_{-1}^{1} f(x) d x \approx & {[f(-1)+f(1)] } \\
& +\beta\left[f^{(1)}(1)-f^{(1)}(-1)\right],
\end{aligned}
$$

where $\vec{a}=(1,1)$ with $n_{0}=1$ and $\vec{a}^{c}=(0,0)$ with $n_{c}=1$. For $1 \leq l \leq n_{\beta}$ the kernel is given by

$$
\begin{aligned}
& K_{\mathrm{Q}, l}(\eta ; 1, \beta)=\frac{(1-\eta)^{l-1}}{(l+1) !}\left[(1-\eta)^{2}-(l+1)(1-\eta)\right. \\
& -l(l+1) \beta] \mathbf{1}_{I^{+}}(\eta)+(-1)^{l+1} \frac{(\eta+1)^{l-1}}{(l+1) !}\left[(\eta+1)^{2}\right. \\
& -(l+1)(\eta+1)-l(l+1) \beta] \mathbf{1}_{I^{-}}(\eta) .
\end{aligned}
$$

Then $C_{l, p}(\beta)=\left\|K_{Q, l}(\cdot ; 1, \beta)\right\|_{q, I}$, where

$$
\left\|K_{\mathrm{Q}, l}(\cdot ; 1, \beta)\right\|_{q, I}= \begin{cases}\frac{1}{(l+1) !}\left[2 \int_{0}^{1}\left[(1-\eta)^{l-1}\left|(1-\eta)^{2}-(l+1)(1-\eta)-l(l+1) \beta\right|\right]^{q} d \eta\right]^{1 / q} & \text { for } 1 \leq q<\infty, \\ \frac{1}{(l+1) !} \max _{\eta \in[0,1]}(1-\eta)^{l-1}\left|(1-\eta)^{2}-(l+1)(1-\eta)-l(l+1) \beta\right| & \text { for } q=\infty .\end{cases}
$$

For this method $R_{1}\left(x^{2} ; 1\right)=-4 / 3$ and $R_{1}^{c}\left(x^{2} ; 1\right)=4$, so $\beta_{*}=-1 / 3$. We obtain $n_{\beta_{*}}=3$, and for any $\beta \neq \beta_{*}$ we have $n_{\beta}=1$. We have computed some constants $C_{l, p}(\beta)$ with $p=1,2,+\infty($ or $q=+\infty, 2,1)$, for $l=1\left(=n_{\beta}\right)$ using $\beta=0 \neq \beta_{*}$ and $\beta_{q}^{*} \neq \beta_{*}$, and for $l=1,2,3$ $\left(=n_{\beta_{*}}\right)$ using $\beta=\beta_{*}=-1 / 3$ as reported in Table 2 . In summary

(i) for $l=1$, we have $C_{1, p}\left(\beta_{q}^{*}\right) \leq C_{1, p}\left(\beta_{*}\right) \leq C_{1, p}(0)$;

(ii) for $\beta_{*}=-1 / 3$, we have $C_{3, p}\left(\beta_{*}\right) \leq C_{2, p}\left(\beta_{*}\right) \leq$ $C_{1, p}\left(\beta_{*}\right)$. 
TABLE 1: Best constants for the midpoint rule.

\begin{tabular}{|c|c|c|c|c|c|c|c|}
\hline \multirow{4}{*}{$p$} & \multirow{4}{*}{$q$} & \multicolumn{6}{|c|}{ Midpoint rule } \\
\hline & & \multirow{2}{*}{\multicolumn{3}{|c|}{$\begin{array}{c}\beta \in \mathbb{R} \backslash\{1 / 6\}, n_{\beta}=1 \\
l=1\end{array}$}} & \multicolumn{3}{|c|}{$\beta_{*}=1 / 6, n_{\beta_{*}}=3$} \\
\hline & & & & & \multirow{2}{*}{$\begin{array}{c}l=1 \\
C_{1, p}\left(\beta_{*}\right)\end{array}$} & \multirow{2}{*}{$\begin{array}{c}l=2 \\
C_{2, p}\left(\beta_{*}\right)\end{array}$} & \multirow{2}{*}{$\begin{array}{c}l=3 \\
C_{3, p}\left(\beta_{*}\right)\end{array}$} \\
\hline & & $C_{1, p}(0)$ & $\beta_{q}^{*}$ & $C_{1, p}\left(\beta_{q}^{*}\right)$ & & & \\
\hline \multirow{2}{*}{1} & \multirow{2}{*}{$\infty$} & 1 & 1 & 1 & 1 & 1 & 1 \\
\hline & & $\overline{2}$ & $\overline{4}$ & $\overline{4}$ & $\overline{3}$ & $9 \sqrt{3}$ & $\overline{24}$ \\
\hline \multirow[b]{2}{*}{2} & & 1 & 1 & $1 \sqrt{2}$ & $1 \sqrt{2}$ & 2 & $1 \longdiv { 1 0 7 }$ \\
\hline & & $\sqrt{10}$ & $\overline{6}$ & $\overline{3} \sqrt{5}$ & $\overline{3} \sqrt{5}$ & $3 \sqrt{105}$ & $\overline{36} \sqrt{70}$ \\
\hline \multirow[b]{2}{*}{$\infty$} & & $\underline{1}$ & 1 & $\underline{1}$ & 4 & 1 & 7 \\
\hline & 1 & $\overline{3}$ & $\overline{8}$ & $\overline{4}$ & $\overline{9 \sqrt{3}}$ & $\overline{12}$ & $\overline{180}$ \\
\hline
\end{tabular}

TABLE 2: Best constants for the trapezoidal rule.

\begin{tabular}{|c|c|c|c|c|c|c|c|}
\hline \multirow{4}{*}{$p$} & \multirow{4}{*}{$q$} & \multicolumn{6}{|c|}{ Trapezoidal rule } \\
\hline & & \multirow{2}{*}{\multicolumn{3}{|c|}{$\begin{array}{c}\beta \in \mathbb{R} \backslash\{-1 / 3\}, n_{\beta}=1 \\
l=1\end{array}$}} & \multicolumn{3}{|c|}{$\beta_{*}=-1 / 3, n_{\beta_{*}}=3$} \\
\hline & & & & & $l=1$ & $l=2$ & $l=3$ \\
\hline & & $C_{1, p}(0)$ & $\beta_{q}^{*}$ & $C_{1, p}\left(\beta_{q}^{*}\right)$ & $C_{1, p}\left(\beta_{*}\right)$ & $C_{2, p}\left(\beta_{*}\right)$ & $C_{3, p}\left(\beta_{*}\right)$ \\
\hline \multirow{2}{*}{1} & \multirow{2}{*}{$\infty$} & 1 & 1 & 1 & 1 & 2 & 1 \\
\hline & & $\overline{2}$ & $\overline{4}$ & $\overline{4}$ & $\overline{3}$ & $\overline{3 \sqrt{3}}$ & $\overline{24}$ \\
\hline \multirow{2}{*}{2} & \multirow[t]{2}{*}{2} & 2 & 1 & $1 \sqrt{2}$ & $1 \sqrt{2}$ & 2 & 2 \\
\hline & & $\overline{\sqrt{15}}$ & $\overline{3}$ & $3 \sqrt{5}$ & $\overline{3} \sqrt{5}$ & $\overline{3 \sqrt{105}}$ & $9 \sqrt{35}$ \\
\hline \multirow{2}{*}{$\infty$} & 1 & 2 & 3 & 1 & 4 & 1 & 2 \\
\hline & 1 & $\overline{3}$ & $\overline{8}$ & $\overline{4}$ & $9 \sqrt{3}$ & $\overline{12}$ & $\overline{45}$ \\
\hline
\end{tabular}

Remark 8. The $\phi(x)$ function considered in [3] corresponds to the Peano kernel $K_{\mathrm{Q}, 1}(\eta ; 1, \beta)$. Also the constants obtained in [3] correspond to the constants we obtained here.

6.3. First Simpson's Rule. Let $n=2$ and $\vec{x}=(-1,0,1)$; then the quadrature formula is

$$
\begin{aligned}
\int_{-1}^{1} f(x) d x \approx & {\left[\frac{1}{3} f(-1)+\frac{4}{3} f(0)+\frac{1}{3} f(1)\right] } \\
& -\beta[2 f(-1)-4 f(0)+2 f(1)] \\
& +\beta\left[f^{(1)}(1)-f^{(1)}(-1)\right]
\end{aligned}
$$

where $\vec{a}=(1 / 3,4 / 3,1 / 3)$ with $n_{0}=3$ and $\vec{a}^{c}=(2,-4,2)$ with $n_{c}=3$. For $1 \leq l \leq n_{\beta}$, the kernel is given by

$$
\begin{aligned}
& K_{\mathrm{Q}, l}(\eta ; 1, \beta)=\frac{(1-\eta)^{l-1}}{(l+1) !}\left[(1-\eta)^{2}\right. \\
& \left.-(l+1)\left(\frac{1}{3}-2 \beta\right)(1-\eta)-l(l+1) \beta\right] \mathbf{1}_{I^{+}}(\eta) \\
& \quad+(-1)^{l+1} \frac{(\eta+1)^{l-1}}{(l+1) !}\left[(\eta+1)^{2}\right. \\
& \left.\quad-(l+1)\left(\frac{1}{3}-2 \beta\right)(\eta+1)-l(l+1) \beta\right] \mathbf{1}_{I^{-}}(\eta)
\end{aligned}
$$

For this method $R_{2}\left(x^{4} ; 1\right)=-4 / 15$ and $R_{2}^{c}\left(x^{4} ; 1\right)=4$, so $\beta_{*}=-1 / 15$. We obtain $n_{\beta_{*}}=5$, and for any $\beta \neq \beta_{*}$ we have
TABLE 3: Values of $C_{3, p}(0)$ for the Simpson's rules.

\begin{tabular}{cccc}
\hline & & \multicolumn{2}{c}{ Simpson's rules, $\beta=0, n_{0}=3$} \\
$p$ & $q$ & $(1 / 3,4 / 3,1 / 3)$ & $(1 / 4,3 / 4,3 / 4,1 / 4)$ \\
& & $C_{3, p}(0)$ & $C_{3, p}(0)$ \\
\hline 1 & $\infty$ & $\frac{1}{72}$ & $\frac{1}{216}$ \\
2 & 2 & $\frac{1}{36 \sqrt{7}}$ & $\frac{1}{81} \sqrt{\frac{13}{105}}$ \\
$\infty$ & 1 & $\frac{1}{90}$ & $\frac{2}{405}$ \\
\hline
\end{tabular}

$n_{\beta}=3$. We have only computed $C_{3, p}(0)=\left\|K_{\mathrm{Q}, 3}(\cdot ; 1,0)\right\|_{q, I}$, for $p=1,2,+\infty$ (or $q=+\infty, 2,1)$, to compare with the midpoint and trapezoidal rules (see Table 3 ). Identifying $\beta_{q}^{*}$ is more complicated.

6.4. Second Simpson's Rule. Let $n=3$ and $\vec{x}=(-1$, $-1 / 3,1 / 3,1)$; then the quadrature formula is

$$
\begin{aligned}
& \int_{-1}^{1} f(x) d x \\
& \approx\left[\frac{1}{4} f(-1)+\frac{3}{4} f\left(-\frac{1}{3}\right)+\frac{3}{4} f\left(\frac{1}{3}\right)+\frac{1}{4} f(1)\right] \\
& \quad-\beta\left[\frac{9}{4} f(-1)-\frac{9}{4} f\left(-\frac{1}{3}\right)-\frac{9}{4} f\left(\frac{1}{3}\right)+\frac{9}{4} f(1)\right] \\
& \quad+\beta\left[f^{(1)}(1)-f^{(1)}(-1)\right],
\end{aligned}
$$

where $\vec{a}=(1 / 4,3 / 4,3 / 4,1 / 4)$ with $n_{0}=3$ and $\vec{a}^{c}=$ $(9 / 4,-9 / 4,-9 / 4,9 / 4)$ with $n_{c}=3$. For $1 \leq l \leq n_{\beta}$ the kernel is given by

$$
\begin{aligned}
& K_{\mathrm{Q}, l}(\eta ; 1, \beta)=\left[\frac { ( 1 - \eta ) ^ { l - 1 } } { ( l + 1 ) ! } \left[(1-\eta)^{2}\right.\right. \\
& \left.-(l+1) \frac{(1-9 \beta)}{4}(1-\eta)-l(l+1) \beta\right] \\
& \left.-\frac{3(l+1)(1+3 \beta)}{4(l+1) !}\left(\frac{1}{3}-\eta\right)_{+}^{l}\right] \mathbf{1}_{I^{+}}(\eta) \\
& +\left[\frac { ( \eta + 1 ) ^ { l - 1 } } { ( l + 1 ) ! } \left[(\eta+1)^{2}-(l+1) \frac{(1-9 \beta)}{4}(\eta+1)\right.\right. \\
& \left.-l(l+1) \beta]-\frac{3(l+1)(1+3 \beta)}{4(l+1) !}\left(\eta+\frac{1}{3}\right)_{+}^{l}\right] \\
& \cdot \mathbf{1}_{I^{-}}(\eta) .
\end{aligned}
$$

For this method $R_{3}\left(x^{4} ; 1\right)=-16 / 135$ and $R_{4}^{c}\left(x^{4} ; 1\right)=$ $32 / 9$, so $\beta_{*}=-1 / 30$. We obtain $n_{\beta_{*}}=5$, and for any $\beta \neq \beta_{*}$ we have $n_{\beta}=3$. We have only computed $C_{3, p}(0)=$ $\left\|K_{Q, 3}(\cdot ; 1,0)\right\|_{q, I}$, for $p=1,2,+\infty$ (or $\left.q=+\infty, 2,1\right)$, to compare with the midpoint and trapezoidal rules (see Table 3). Identifying $\beta_{q}^{*}$ is more complicated. 
TABLE 4: Results for the midpoint rule.

\begin{tabular}{|c|c|c|c|c|}
\hline \multicolumn{5}{|c|}{ Midpoint rule } \\
\hline \multirow{3}{*}{$M$} & \multicolumn{4}{|c|}{ Standard rule $\left(\beta=0 ; n_{0}=1\right)$} \\
\hline & Computed & Absolute error & Relative error & Estimated order \\
\hline & integral & $\operatorname{ABS}(M)$ & $\operatorname{REL}(M)$ & $\mathrm{EO}(M) \approx n_{0}+1$ \\
\hline 5 & 1.4527054409211020 & 0.0062641086729669 & $4.33 e-03$ & \\
\hline 10 & 1.4479877945462096 & 0.0015464622980745 & $1.07 e-03$ & 2.0 \\
\hline 15 & 1.4471270587692653 & 0.0006857265211302 & $4.74 e-04$ & 2.0 \\
\hline 20 & 1.4468267417338132 & 0.0003854094856781 & $2.66 e-04$ & 2.0 \\
\hline \multirow[t]{2}{*}{25} & 1.4466879021519083 & 0.0002465699037733 & $1.70 e-04$ & 2.0 \\
\hline & \multicolumn{4}{|c|}{ Corrected rule $\left(\beta=\beta_{*}=1 / 6 ; n_{\beta_{*}}=3\right)$} \\
\hline \multirow[t]{2}{*}{$M$} & Computed & Absolute error & Relative error & Estimated order \\
\hline & integral & $\operatorname{ABS}(M)$ & $\operatorname{REL}(M)$ & $\mathrm{EO}(M) \approx n_{\beta_{*}}+1$ \\
\hline 5 & 1.4465452831301751 & 0.0001039508820400 & $7.19 e-05$ & \\
\hline 10 & 1.4464477550984778 & 0.0000064228503427 & $4.44 e-06$ & 4.0 \\
\hline 15 & 1.4464425967924956 & 0.0000012645443606 & $8.74 e-07$ & 4.0 \\
\hline 20 & 1.4464417318718803 & 0.0000003996237452 & $2.76 e-07$ & 4.0 \\
\hline 25 & 1.4464414958402714 & 0.0000001635921363 & $1.13 e-07$ & 4.0 \\
\hline
\end{tabular}

TABLE 5: Results for the trapezoidal rule.

\begin{tabular}{|c|c|c|c|c|}
\hline \multicolumn{5}{|c|}{ Trapezoidal rule } \\
\hline \multirow{3}{*}{$M$} & \multicolumn{4}{|c|}{ Standard rule $\left(\beta=0 ; n_{0}=1\right)$} \\
\hline & Computed & Absolute error & Relative error & Estimated order \\
\hline & integral & $\operatorname{ABS}(M)$ & $\operatorname{REL}(M)$ & $\mathrm{EO}(M) \approx n_{0}+1$ \\
\hline 5 & 1.4340023935151260 & 0.0124389387330091 & $8.60 e-03$ & \\
\hline 10 & 1.4433539172181140 & 0.0030874150300211 & $2.13 e-03$ & 2.0 \\
\hline 15 & 1.4450709634929373 & 0.0013703687551978 & $9.47 e-04$ & 2.0 \\
\hline 20 & 1.4456708558821618 & 0.0007704763659733 & $5.33 e-04$ & 2.0 \\
\hline \multirow[t]{2}{*}{25} & 1.4459483326810811 & 0.0004929995670540 & $3.41 e-04$ & 2.0 \\
\hline & \multicolumn{4}{|c|}{ Corrected rule $\left(\beta=\beta_{*}=-1 / 3 ; n_{\beta_{*}}=3\right)$} \\
\hline \multirow[t]{2}{*}{$M$} & Computed & Absolute error & Relative error & Estimated order \\
\hline & integral & $\operatorname{ABS}(M)$ & $\operatorname{REL}(M)$ & $\mathrm{EO}(M) \approx n_{\beta_{*}}+1$ \\
\hline 5 & 1.4463227090969801 & 0.0001186231511550 & $8.20 e-05$ & \\
\hline 10 & 1.4464339961135775 & 0.0000073361345576 & $5.07 e-06$ & 4.0 \\
\hline 15 & 1.4464398874464766 & 0.0000014448016585 & $9.99 e-07$ & 4.0 \\
\hline 20 & 1.4464408756060276 & 0.0000004566421075 & $3.16 e-07$ & 4.0 \\
\hline 25 & 1.4464411453043553 & 0.0000001869437798 & $1.29 e-07$ & 4.0 \\
\hline
\end{tabular}

6.5. Discussion. Let us observe that the constants of Table 1 (for the midpoint rule) are all equal or less than the constants of Table 2 (for the trapezoidal rule). Moreover, $C_{3, p}(0)$ for the second (noncorrected, $\beta=0$ ) Simpson rule are all less than the $C_{3, p}(0)$ of the first (noncorrected, $\beta=0$ ) Simpson rule which are themselves less than the $C_{3, p}\left(\beta_{*}\right)$ of the corrected midpoint rule.

\section{Corrected Composite Quadrature Rules}

7.1. The Method. For an integral $\int_{a}^{b} f(\xi) d \xi$, where $f \in$ $A C^{l+1, p}([a, b])$, a composite rule uses a partition of $[a, b]$ in $M$ subintervals and applies a formula on each subinterval.
To simplify here we consider subintervals of equal length $h=(b-a) / 2 M$. Let us set $\xi_{m}=a+2 m h$ for $m=0, \ldots, M$ and $c_{m}=\left(\xi_{m-1}+\xi_{m}\right) / 2$ for $m=1, \ldots, M$. Then

$$
\begin{aligned}
\int_{a}^{b} f(\xi) d \xi & =\sum_{m=1}^{M} \int_{\xi_{m-1}}^{\xi_{m}} f(\xi) d \xi \\
& =\sum_{m=1}^{M} \int_{-h}^{h} f\left(c_{m}+x\right) d x \\
& =h \sum_{m=1}^{M} Q\left(f\left(c_{m}+\cdot\right) ; h\right) .
\end{aligned}
$$


TABLE 6: Results for the first Simpson rule.

\begin{tabular}{|c|c|c|c|c|}
\hline \multicolumn{5}{|c|}{ First Simpson rule } \\
\hline \multirow{3}{*}{$M$} & \multicolumn{4}{|c|}{ Standard rule $\left(\beta=0 ; n_{0}=3\right)$} \\
\hline & Computed & Absolute error & Relative error & Estimated order \\
\hline & integral & $\operatorname{ABS}(M)$ & $\operatorname{REL}(M)$ & $\mathrm{EO}(M) \approx n_{0}+1$ \\
\hline 5 & 1.4464710917857768 & 0.0000297595376417 & $2.06 e-05$ & \\
\hline 10 & 1.4464431687701778 & 0.0000018365220427 & $1.27 e-06$ & 4.0 \\
\hline 15 & 1.4464416936771560 & 0.0000003614290209 & $2.50 e-07$ & 4.0 \\
\hline 20 & 1.4464414464499289 & 0.0000001142017938 & $7.90 e-08$ & 4.0 \\
\hline \multirow[t]{2}{*}{25} & 1.4464413789949659 & 0.0000000467468309 & $3.23 e-08$ & 4.0 \\
\hline & \multicolumn{4}{|c|}{ Corrected rule $\left(\beta=\beta_{*}=-1 / 15 ; n_{\beta_{*}}=5\right)$} \\
\hline \multirow[t]{2}{*}{$M$} & Computed & Absolute error & Relative error & Estimated order \\
\hline & integral & $\operatorname{ABS}(M)$ & $\operatorname{REL}(M)$ & $\mathrm{EO}(M) \approx n_{\beta_{*}}+1$ \\
\hline 5 & 1.4464414152480176 & 0.0000000829998825 & $5.74 e-08$ & \\
\hline 10 & 1.4464413342388578 & 0.0000000019907227 & $1.38 e-09$ & 5.4 \\
\hline 15 & 1.4464413324310201 & 0.0000000001828850 & $1.26 e-10$ & 5.9 \\
\hline 20 & 1.4464413322811487 & 0.0000000000330136 & $2.28 e-11$ & 6.0 \\
\hline 25 & 1.4464413322568439 & 0.0000000000087088 & $6.02 e-12$ & 6.0 \\
\hline
\end{tabular}

TABLE 7: Results for the second Simpson rule.

\begin{tabular}{|c|c|c|c|c|}
\hline \multicolumn{5}{|c|}{ Second Simpson rule } \\
\hline \multirow{3}{*}{$M$} & \multicolumn{4}{|c|}{ Standard rule $\left(\beta=0 ; n_{0}=3\right)$} \\
\hline & Computed & Absolute error & Relative error & Estimated order \\
\hline & integral & $\operatorname{ABS}(M)$ & $\operatorname{REL}(M)$ & $\mathrm{EO}(M) \approx n_{0}+1$ \\
\hline 5 & 1.4464545347401641 & 0.0000132024920290 & $9.13 e-06$ & \\
\hline 10 & 1.4464421478702252 & 0.0000008156220901 & $5.64 e-07$ & 4.0 \\
\hline 15 & 1.4464414928269707 & 0.0000001605788356 & $1.11 e-07$ & 4.0 \\
\hline 20 & 1.4464413829943144 & 0.0000000507461793 & $3.51 e-08$ & 4.0 \\
\hline \multirow[t]{2}{*}{25} & 1.4464413530218192 & 0.0000000207736841 & $1.44 e-08$ & 4.0 \\
\hline & \multicolumn{4}{|c|}{ Corrected rule $\left(\beta=\beta_{*}=-1 / 30 ; n_{\beta_{*}}=5\right)$} \\
\hline \multirow[t]{2}{*}{$M$} & Computed & Absolute error & Relative error & Estimated order \\
\hline & integral & $\operatorname{ABS}(M)$ & $\operatorname{REL}(M)$ & $\mathrm{EO}(M) \approx n_{\beta_{*}}+1$ \\
\hline 5 & 1.4464413521758457 & 0.0000000199277106 & $1.38 e-08$ & \\
\hline 10 & 1.4464413326945604 & 0.0000000004464253 & $3.09 e-10$ & 5.5 \\
\hline 15 & 1.4464413322889214 & 0.0000000000407863 & $2.82 e-11$ & 5.9 \\
\hline 20 & 1.4464413322554857 & 0.0000000000073506 & $5.08 e-12$ & 6.0 \\
\hline 25 & 1.4464413322500729 & 0.0000000000019378 & $1.34 e-12$ & 6.0 \\
\hline
\end{tabular}

The corrected composite rule is then defined by

$$
\begin{aligned}
& C_{n}(f ; \beta) \\
& =h \sum_{m=1}^{M} \widetilde{Q}_{n}\left(f\left(c_{m}+\cdot\right) ; h, \beta\right) \\
& =h \sum_{m=1}^{M}\left[Q_{n}\left(f\left(c_{m}+\cdot\right) ; h\right)+Q_{\beta}\left(f\left(c_{m}+\cdot\right) ; h\right)\right] \\
& =h \sum_{m=1}^{M} Q_{n}\left(f\left(c_{m}+\cdot\right) ; h\right) \\
& \quad+\beta h^{2}\left[f^{(1)}(b)-f^{(1)}(a)\right]
\end{aligned}
$$

since there are cancellations of the first derivatives at the endpoints of the subintervals. The truncation error is

$$
\int_{a}^{b} f(\xi) d \xi-C Q_{n}(f ; \beta)=h \sum_{m=1}^{M} R_{n}\left(f\left(c_{m}+\cdot\right) ; h, \beta\right) .
$$

But

$$
\begin{aligned}
& \left|\sum_{m=1}^{M} R_{n}\left(f\left(c_{m}+\cdot\right) ; h, \beta\right)\right| \\
& \quad \leq \sum_{m=1}^{M}\left|R_{n}\left(f\left(c_{m}+\cdot\right) ; h, \beta\right)\right| \\
& \quad \leq h^{l+1-(1 / p)} C_{l, p}(\beta) \sum_{m=1}^{M}\left\|f^{(l+1)}\right\|_{p, c_{m}+I_{h}}
\end{aligned}
$$




$$
\begin{aligned}
& \leq h^{l+1-(1 / p)} M^{1-(1 / p)} C_{l, p}(\beta)\left\|f^{(l+1)}\right\|_{p,[a, b]} \\
& \leq h^{l}\left(\frac{b-a}{2}\right)^{1-(1 / p)} C_{l, p}(\beta)\left\|f^{(l+1)}\right\|_{p,[a, b]} .
\end{aligned}
$$

[5] L. L. Schumaker, Spline Functions: Basic Theory, Wiley, New York, NY, USA, 1981.

[6] R. A. Adams, Sobolev Spaces, Academic Press, New York, NY, USA, 1975.

It follows that

$$
\begin{aligned}
& \left|\int_{a}^{b} f(\xi) d \xi-C Q_{n}(f ; \beta)\right| \\
& \quad \leq\left(\frac{b-a}{2 M}\right)^{l+1}\left(\frac{b-a}{2}\right)^{1-(1 / p)} C_{l, p}(\beta)\left\|f^{(l+1)}\right\|_{p,[a, b]}
\end{aligned}
$$

for $l=1, \ldots, n_{\beta}$ and $f \in A C^{l+1, p}([a, b])$.

7.2. Numerical Examples. To illustrate the results, we approximate the following integral:

$$
\begin{aligned}
\int_{-0.5}^{1.5} \frac{d x}{1+x^{2}} & =\arctan (1.5)-\arctan (-0.5) \\
& =1.4464413322481351 \ldots
\end{aligned}
$$

using (55) for $\beta=0$ and for $\beta=\beta_{*}$. Since for the absolute error, noted as $\operatorname{ABS}(M)$, we have from (58)

$$
\left|\int_{a}^{b} f(\xi) d \xi-C Q_{n}(\tilde{f} ; \beta)\right|=\operatorname{ABS}(M) \approx O\left(\frac{1}{M^{l+1}}\right),
$$

the order $l+1$ is estimated by the formula

$$
\mathrm{EO}(M)=\ln \left(\frac{\mathrm{ABS}(M-5)}{\mathrm{ABS}(M)}\right) \div \ln \left(\frac{M}{M-5}\right)
$$

for $M=10,15,20,25$, in Tables 4, 5, 6, and 7. Because the function we integrate is regular, we can identify the maximal orders $n_{0}+1$ and $n_{\beta_{*}}+1$ which correspond to the theoretical results.

\section{Conflict of Interests}

The author declares that there is no conflict of interests regarding the publication of this paper.

\section{References}

[1] P. J. Davis and P. Rabinowitz, Methods of Numerical Integration, Computer Science and Applied Mathematics, Academic Press, Orlando, Fla, USA, 2nd edition, 1984.

[2] Z. Liu, "Error estimates for some composite corrected quadrature rules," Applied Mathematics Letters, vol. 22, no. 5, pp. 771775, 2009.

[3] E. Talvila and M. Wiersma, "Optimal error estimates for corrected trapezoidal rules," Journal of Mathematical Inequalities, vol. 6, no. 3, pp. 431-445, 2012.

[4] E. Asplund and L. Bungart, A First Course in Integration, Holt, Rinehart and Winston, New York, NY, USA, 1966. 


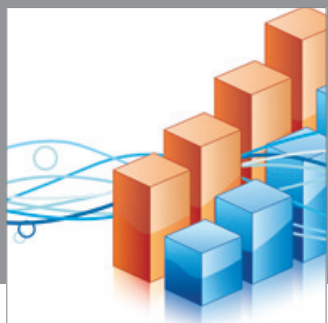

Advances in

Operations Research

mansans

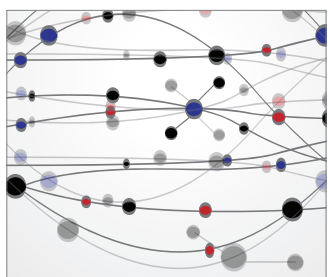

The Scientific World Journal
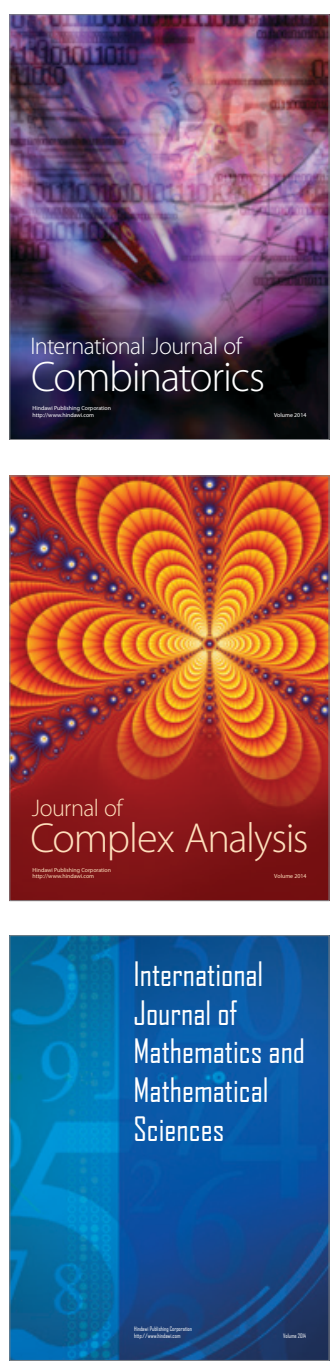
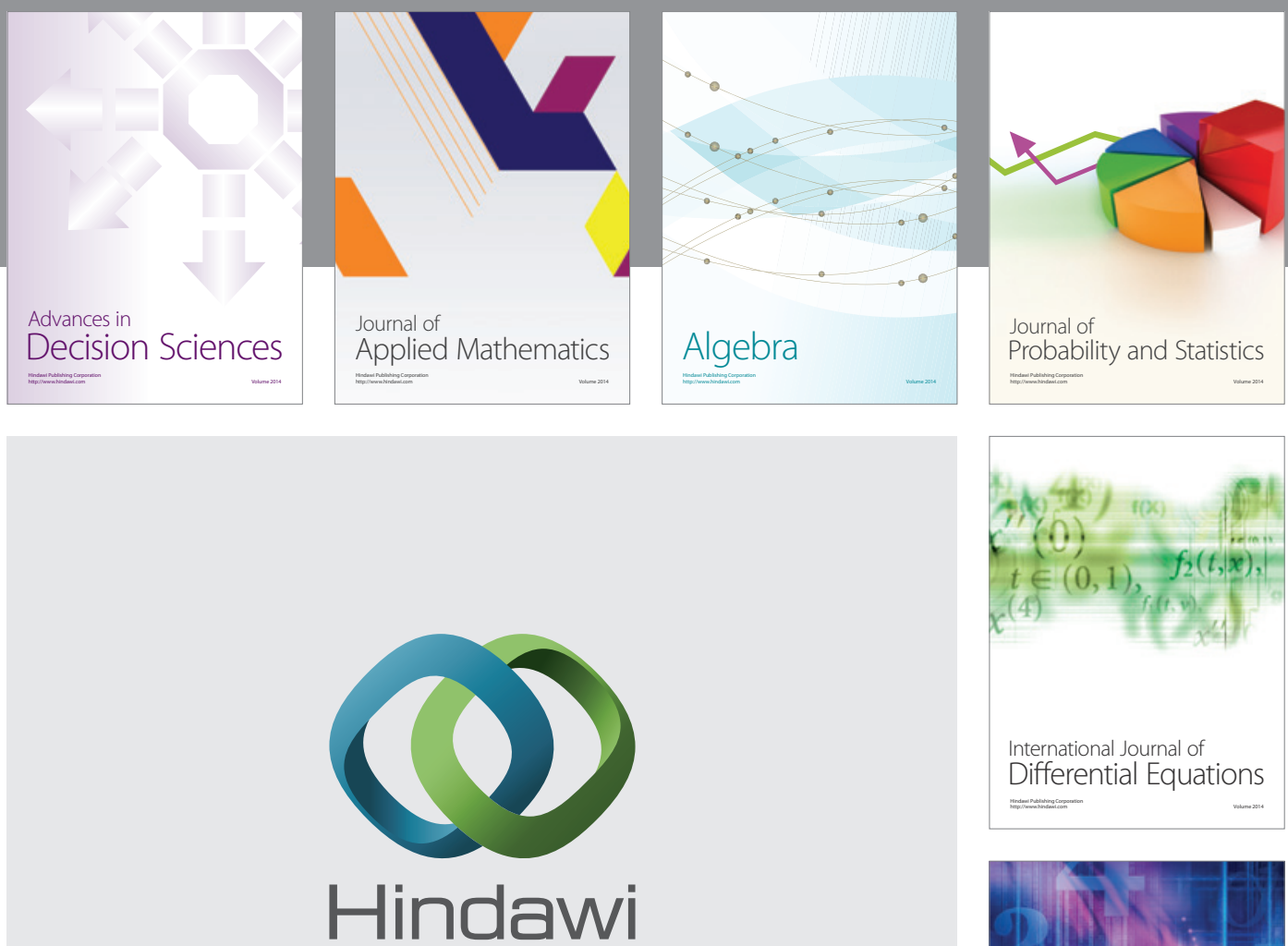

Submit your manuscripts at http://www.hindawi.com
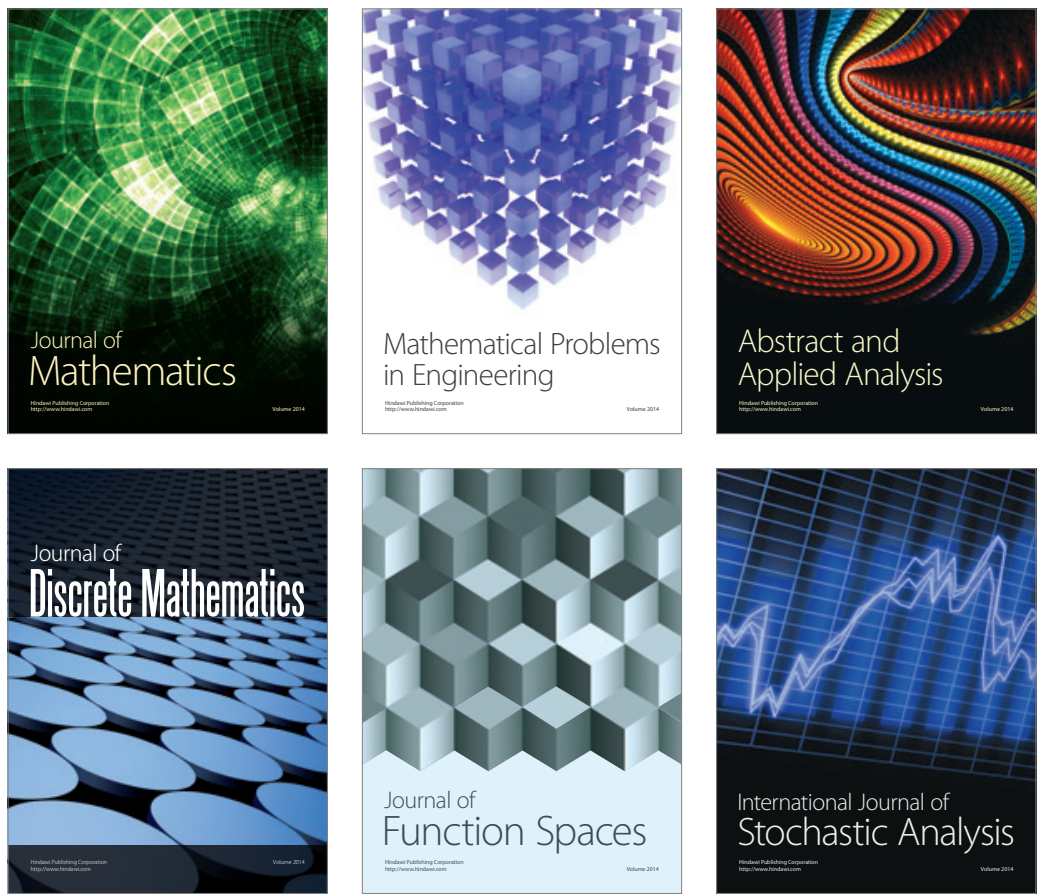

Journal of

Function Spaces

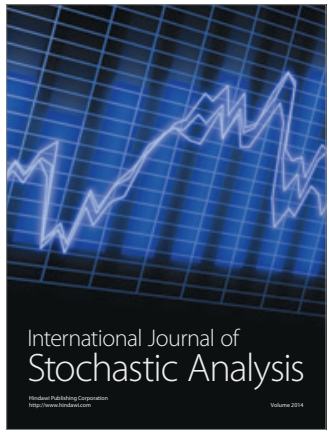

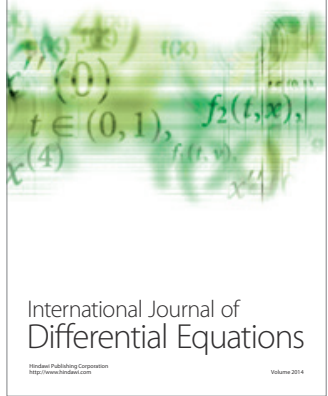
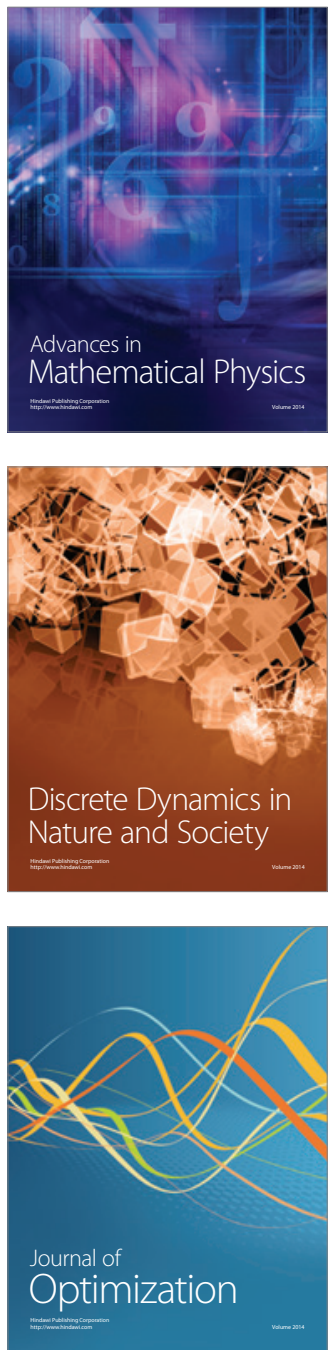